\title{
SOME TESTS FOR WHETHER A NARROW NEUTRAL RESONANCE CAN BE A HIGGS PARTICLE 岤
}

\author{
Howard E. HABER \\ Department of Physics, University of California, Santa Cruz, CA 95064, USA ${ }^{1}$ \\ and Stanford Linear Accelerator Center, Stanford, CA 94305, USA \\ and \\ G.L. KANE \\ Randall Laboratory of Physics, University of Michigan, Ann Arbor, MI 48109, USA
}

Received 26 September 1983

Revised manuscript received 7 November 1983

We consider under what conditions any narrow neutral resonance, particularly the recently reported state $\xi(2.2)$, could be a Higgs particle, either a fundamental boson or a composite technicolor state. A number of tests are summarized including what is expected in $\Upsilon$ decay, b-quark decay, $Z^{0}$ decays, branching ratios and properties of $\xi(2.2)$ decay, and produc tion at hadron machines. Implications for the standard model, technicolor, and supersymmetry are discussed.

1. Introduction. As is well known, the Higgs sector of the standard model [1] is not understood. Fundamental or composite Higgs particles could exist at any mass; some new physics must exist, but there need not be any light particles. (For recent reviews, see ref. [2].)

If physical Higgs particles exist, some of their properties are uniquely determined, and others have several alternatives. They must have spin zero, but they can be scalar or pseudoscalar. In the simplest model, with a single Higgs doublet, the Higgs must be a scalar and the Higgs - fermion coupling must be proportional to the fermion mass since this coupling is the source of the mass term, and one expects $\Gamma \lesssim 10 \mathrm{eV}$ for $m_{\mathrm{H}} \sim 1 \mathrm{GeV}$ and $m_{\mathrm{f}} \sim 100 \mathrm{MeV}$. That is, for Higgs masses in the $\mathrm{GeV}$ range, the Higgs width will be far narrower than the resolution of normal particle physics experiments.

Consequently, any newly discovered resonance

\footnotetext{
Work supported in part by the Department of Energy, contracts DE-AC03-76SF00515 and DE-AC02-76ER01112 and NSF grant PHY 8115541-02.

${ }^{1}$ Permanent address.
}

whose width is narrower than the experimental resolution should be considered as a possible candidate for a Higgs, and should be studied in that context. ${ }^{\neq 1}$ Higgs physics is too important to not take any possible instance seriously, even if it may not be a likely one. In this paper we carry out that analysis for the recently reported [3] state $\xi(2.2)$, whose width is presently consistent with experimental resolution. Clearly every effort should be made to detect a small width - with present experimental limitations perhaps a width as small as $10 \mathrm{MeV}$ could be detected. In addition, it is obvious that the spin measurement is also very crucial.

The reported [3] data are the observation by the Mark III collaboration of a process $\psi \rightarrow \gamma+\xi(2.2)$, $\xi(2.2) \rightarrow \mathrm{K}^{+} \mathrm{K}^{-}, \mathrm{K}_{\mathrm{S}} \mathrm{K}_{\mathrm{S}}$, with $m_{\xi}=2.22 \pm 0.02 \mathrm{GeV}$, $\Gamma_{\xi}=30 \pm 10 \pm 20 \mathrm{MeV}$, and BR $(\psi \rightarrow \gamma+\xi) \mathrm{BR}(\xi \rightarrow$ $\left.\mathrm{K}^{+} \mathrm{K}^{-}\right)=(8.0 \pm 2.0 \pm 1.6) \times 10^{-5}$. This branching

${ }^{\neq 1}$ Experimentally, it should be easy to distinguish a light Higgs boson from ordinary strongly interacting scalar states. Apart from its extremely narrow width, the Higgs boson would be distinguished by having a non-negligible branching ratio into leptons (either $\mu^{+} \mu^{-}$or $\tau^{+} \tau^{-}$depending on the Higgs mass). 
ratio already implies the $\xi(2.2)$ cannot be the Higgs boson of the standard model for a single Higgs doublet. If there is only one Higgs doublet, the decay rate for $\psi \rightarrow \gamma \mathrm{H}^{0}$ is computable [4], giving

$$
\frac{\Gamma\left(\psi \rightarrow \gamma \mathrm{H}^{0}\right)}{\Gamma\left(\psi \rightarrow \mu^{+} \mu^{-}\right)}=\frac{G_{\mathrm{F}} m_{\psi}^{2}}{4 \sqrt{2} \pi \alpha}\left(1-\frac{m_{\mathrm{H}}^{2}}{m_{\psi}^{2}}\right) \text {. }
$$

Then $\operatorname{BR}\left(\psi \rightarrow \gamma \mathrm{H}^{0}\right) \simeq(3.1 \pm 0.5) \times 10^{-5}$, the error is from the error in $\operatorname{BR}\left(\psi \rightarrow \mu^{+} \mu^{-}\right)$. Assuming the decay of an $\mathrm{H}^{0}$ is dominantly to $\bar{s}$, one expects the decay products to consist of $\mathrm{K}^{+} \mathrm{K}^{-}, \mathrm{K}^{0} \mathrm{~K}^{0}, \mathrm{~K}^{*} \mathrm{~K}^{*}$ (for the latter mode, we expect a factor of 3 enhancement for spin counting for each of the two charge combinations and a factor of about $2 / 3$ for phase space), so it is conservative to take $\mathrm{BR}\left(\mathrm{H}^{0} \rightarrow \mathrm{K}^{+} \mathrm{K}^{-}\right) \leqslant 1 / 6$. Then for $m_{\mathrm{H}}=2.2 \mathrm{GeV}, \mathrm{BR}\left(\psi \rightarrow \gamma \mathrm{H}^{0}\right) \mathrm{BR}\left(\mathrm{H}^{0} \rightarrow \mathrm{K}^{+} \mathrm{K}^{-}\right) \leqslant$ $(0.5 \pm 0.1) \times 10^{-5}$. This is at least an order of magnitude below the reported data, though only at the level of a few standard deviations.

An additional argument against a single $\mathrm{H}^{0}$ interpretation is that $\xi(2.2)$ is considerably lighter than the minimum mass allowed by Linde-Weinberg bound [5]. This bound is obtained by requiring that the spontaneously broken vacuum be lower in energy than the symmetric vacuum when one-loop radiative effects are taken into account. If we define

$$
C=\frac{1}{64 \pi^{2} v^{4}} \sum\left(3 m_{\mathrm{V}}^{4}+m_{\mathrm{H}}^{4}-4 m_{\mathrm{f}}^{4}\right),
$$

summing over the tree-level masses of all species of vector bosons $\mathrm{V}$, Higgs bosons $\mathrm{H}$ and fermions $\mathrm{f}$ (and $v=2^{-1 / 4} G_{\mathrm{F}}^{-1 / 2} \approx 250 \mathrm{GeV}$ ), the Linde-Weinberg bound states that in the one-Higgs doublet model $m_{\mathrm{H}} \geqslant 2 v C^{1 / 2}$. If the vector boson masses dominate eq. (2), then $m_{\mathrm{H}} \geqslant 7 \mathrm{GeV} \neq 2$.

Neither of the above arguments applies to ap-

${ }^{+2}$ Even in the one-Higgs doublet model, there is a way to evade the Linde-Weinberg bound. Linde in fact argued (ref. [5]) that if we lived in a metastable vacuum with lifetime greater than the age of the universe, the Higgs boson mass could be as small as $0.26 \mathrm{GeV}$. However, it is hard to imagine a scenario for the early universe which would result in us being in such a vacuum today. Note that if there exists a very heavy fermion so that $C \approx 0$ [see eq. (2)], then the Linde-Weinberg bound becomes irrelevant. proaches where two or more Higgs doublets are present. Then the Linde-Weinberg bound applies to only one of the scalar Higgs bosons, so only one of the several neutral states need be heavier than $7 \mathrm{GeV}$. The expected branching ratios for $\mathrm{V} \rightarrow \gamma \mathrm{H}^{0}$ can be enhanced [6] by the squares of ratios of vacuum expectation values (VEV) which will be present because there is more than one Higgs to get a VEV.

One can systematically examine models with two or more Higgs doublets [6-8]. There is also theoretical motivation for studying such models. First, at least two Higgs doublets are required in supersymmetric theories. Second, the low energy spectrum of technicolor has many features in common with that of a two-doublet model. One can view supersymmetry and technicolor as two complementary approaches to incorporating Higgs physics into the structure of gauge theories. The former has fundamental scalars which are related to fermions by the supersymmetry; the latter assumes new fundamental fermions rather than new bosons, and obtains bosons as composites.

Therefore, let us see if the $\xi(2.2)$ could fit into an approach with (at least) two Higgs doublets. First, we can conclude that if the $\xi(2.2)$ is to be interpreted as a Higgs boson, one needs an enhancement of the ratio of vacuum expectation values which enters into the coupling to charmed quarks by a factor slightly above 3 . This would lead to a prediction for $\mathrm{BR}(\psi \rightarrow$ $\gamma \mathrm{H}^{0}$ ) of about a factor of 10 larger than that given in eq. (1), consistent with the $\xi(2.2)$ rate. This has several implications for $b$ and $\Upsilon$ decays which we will explore below. In arbitrary two-Higgs models or in supersymmetric theories, such a ratio of VEV's can be quite natural. In technicolor theories of the simplest sort there is only one scale, but in more complicated versions the couplings to ordinary fermions result from a mass matrix diagonalization which can introduce factors that change couplings by the needed amounts. Thus on the basis of the presently available information, $\xi(2.2)$ is a viable candidate for a Higgs particle in any approach beyond the standard model with a single doublet.

Most of our analysis, apart from specifics due to the observation of $\xi(2.2)$ decay into $\mathrm{K}_{\mathrm{S}} \mathrm{K}_{\mathrm{S}}$, applies very generally to any present or future candidate for a Higgs particle in the mass range below about 10 $\mathrm{GeV}$. A summary of this analysis as applied to the $\xi(2.2)$ is given in table 1 . 
Table 1

We summarize the results expected for $\xi(2.2)$ decay and production if it were a Higgs boson. We work in a multi-Higgs doublet extension of the minimal electroweak model, since the observed rate for $\xi \rightarrow K^{+} K^{-}$rules out the possibility that $\xi$ is the Higgs boson of the minimal model.

\begin{tabular}{|c|c|c|}
\hline & & Comments \\
\hline Decays of $\xi$ & $\left.\begin{array}{l}\mathrm{K}_{\mathrm{S}^{\mathrm{K}}} \mathrm{K}_{\mathrm{S}} \\
\mathrm{K}^{+} \mathrm{K}^{-} \\
\mathrm{K}^{*} \overline{\mathrm{K}} \\
\mathrm{K}^{*} \overline{\mathrm{K}}^{*} \\
\mu^{+} \mu^{-} \\
\pi^{+} \pi^{-} \\
\mathrm{e}^{+} \mathrm{e}^{-} \\
\gamma \gamma \\
\rho \rho \\
\phi \phi \\
\eta \eta\end{array}\right\}$ & $\begin{array}{l}\text { seen (implies } J^{P C}=0^{++}, 2^{++}, \ldots \text { ) } \\
\text { seen } \\
\text { would require negative parity admixture } \\
\text { should be seen; can test for parity mix ture } \\
\text { BR } \sim 4-16 \% \\
\text { BR should be very small } \\
\text { BR }(\phi \phi) / \mathrm{BR}\left(\mathrm{K}^{*} \overline{\mathrm{K}}^{*}\right) \sim 10-20 \% \\
\mathrm{BR} \text { should be a few percent }\end{array}$ \\
\hline Production of $\xi$ & $\begin{array}{l}\psi \rightarrow \gamma \xi \\
\Gamma, \Gamma^{\prime}, \Gamma^{\prime \prime} \rightarrow \gamma \xi \\
b \rightarrow s \xi \\
Z^{0} \rightarrow \mathrm{Z}^{0} \xi \\
\mathrm{W}^{ \pm} \rightarrow \mathrm{W}^{ \pm} \xi \\
\mathrm{Z}^{0} \rightarrow \mathrm{H}^{0} \xi \\
\mathrm{KN} \rightarrow \xi \mathrm{X} \\
\mathrm{p} \overline{\mathrm{p}} \rightarrow \psi \rightarrow \gamma \xi\end{array}$ & $\begin{array}{l}\text { requires a ratio of vacuum expectation values of order } \sqrt{10} \\
\text { could be enhanced or suppressed by a factor of } 10 \text { depending on type } \\
\text { of multi-Higgs model } \\
\mathrm{BR} \text { probably large (perhaps as large as } 50 \% \text { ) and thus observable in } \\
\mathrm{B} \rightarrow \mathrm{KKKX}, \mathrm{K}^{+} \mu^{-} \mathrm{X} \text {; may be directly excluded } \\
\mathrm{BR} \sim 1 \% \text { (due to small } \xi \text { mass, the second } \mathrm{Z}^{0} \text {, } \\
\mathrm{W}^{+} \text {is nearly on-shell) } \\
\mathrm{BR} \leqslant 9 \% \text { if one Higgs is scalar and the other is pseudoscalar } \\
\text { signal-to-noise could be } 1: 1 \text { or better for } \mathrm{K}_{\mathrm{S}} \mathrm{K}_{\mathrm{S}} \text { or } \mu^{+} \mu^{-} \text {modes } \\
\text { could get good rate at LEAR }\end{array}$ \\
\hline
\end{tabular}

2. Decays of $\xi(2.2)$. If $\xi(2.2)$ is a Higgs particle one can perform several tests, in addition to the spin and width measurements mentioned above.

(a) Since the couplings should be proportional to masses, one expects

$\Gamma\left(\xi \rightarrow \mu^{+} \mu^{-}\right) / \Gamma_{\text {TOT }} \simeq \frac{1}{3} m_{\mu}^{2} / m_{\mathrm{s}}^{2}$.

The $1 / 3$ is a color factor. Since the current quark mass $m_{\mathrm{s}}$ ranges from about $150 \mathrm{MeV}$ to about 300 $\mathrm{MeV}$, one expects a branching ratio from about $4 \%$ to $16 \%$. Observation of a $\mu^{+} \mu^{-}$signal would make the Higgs interpretation a rather likely one. However, in complicated models it is technically possible to construct couplings which are not strictly proportional to masses, so the absence of a $\mu^{+} \mu^{-}$signal does not rigorously exclude the Higgs interpretation.

(b) If the couplings are proportional to masses, a number of modes should be very small, such as $\mathrm{e}^{+} \mathrm{e}^{-}$, $\gamma \gamma, \mathrm{u} \overline{\mathrm{u}}, \mathrm{d} \overline{\mathrm{d}}$. The last two show up as $\pi \pi$ or $\rho \rho$ modes, which should then not appear. A $\phi \phi$ mode should appear with a ratio of $10-20 \%$ to $\mathrm{K}^{*} \overline{\mathrm{K}}^{*}$ (determined by the probability of getting an $s \bar{s}$ or a d d out of the vacuum and by the phase space suppression.) Also, an $\eta \eta$ mode should be observable.

(c) Information on spin and parity can be obtained by comparing $\mathrm{K}_{\mathrm{S}} \mathrm{K}_{\mathrm{S}}$ (which is observed [3] and requires $J^{P C}=0^{++}, 2^{++}, \ldots$ ), $\mathrm{K}^{+} \mathrm{K}^{-}$(which could require spin $\geqslant 2$ if the decay distribution is not flat), $\mathrm{K}^{*} \overline{\mathrm{K}}$ (which would require odd parity), and $\mathrm{K}^{*} \overline{\mathrm{K}}^{*}$ (whose branching ratio should be somewhat greater than $\mathrm{K} \overline{\mathrm{K}}$ due to spin counting). One can determine the parity of the $\mathrm{K}^{*} \overline{\mathrm{K}}^{*}$ state by comparing [9] the $\mathrm{K}^{*}$ and $\overline{\mathrm{K}}^{*}$ polarizations, which tend to be perpendicular for the pseudoscalar case and parallel for the scalar case. This leads to a correlation for the azimuthal angle between the two $K^{*} \rightarrow K \pi$ decay planes. It is possible (see discussion below) that the Higgs particle is not a definite parity state, so it is very important to look in $\mathrm{K}^{*} \overline{\mathbf{K}}$ and $\mathrm{K}^{*} \overline{\mathrm{K}}^{*}$ for signs of a parity mixture - such a signal would guarantee for a spin zero particle that one is not seeing a strong decay, and would make $\xi(2.2)$ a very strong candidate for a Higgs particle. 
3. Production of $\xi(2.2)$. There are several other ways a Higgs candidate with mass below $10 \mathrm{GeV}$ could be detected immediately or in the relatively near future.

(a) The neutral flavor-changing decay $b \rightarrow \mathrm{s}+\mathrm{H}^{0}$ is expected to have a large branching ratio. It has been calculated rather generally in models with two Higgs doublets by Hall and Wise [10]. The answer depends on several things. ${ }^{\ddagger 3}$ One is the ratio of VEV's discussed above, which we denote by $\eta$. If the $\xi(2.2)$ is identified as the $\mathrm{H}^{0}$, then we can take this ratio as known (i.e. $\eta^{2} \approx 10$ ). The answer also depends on $m_{\mathfrak{t}}$ and on the mass of the charged Higgs which is always present when two or more Higgs doublets exist. For a large range of parameters and $\eta^{2} \approx 10$, the branching ratio for $\mathrm{b} \rightarrow \mathrm{sH}^{0}$ is on the order of $50 \%$ (!), so one would expect a very large rate for $b \rightarrow 3$ strange particles, and a branching ratio of order $5 \%$ for $b \rightarrow s \mu^{+} \mu^{-}$. These numbers hold for typical $m_{\mathrm{t}} \gtrsim 20 \mathrm{GeV}$, except that for a narrow region where $m_{\mathrm{H}^{ \pm}} \simeq 5 m_{\mathrm{t}} \gtrsim m_{\mathrm{W}}$ a cancellation occurs and the rate can be considerably suppressed. Thus, observation of $\xi(2.2)$ in b decay would be strong evidence in favor of a Higgs interpretation, while non-observation is evidence either against the Higgs interpretation or possibly implies

$m_{\mathrm{H}^{+}} / m_{\mathrm{t}} \simeq 5$. Recent data [11] from CLEO quotes an upper limit of $\mathrm{BR}\left(\mathrm{B} \rightarrow \mathrm{X} \mu^{+} \mu^{-}\right)<0.3 \%$ (at $90 \% \mathrm{CL}$ ) which makes the Higgs interpretation rather problematical. ${ }^{\ddagger 4}$

(b) If $\psi \rightarrow \gamma \mathrm{H}^{0}$, so will $\Upsilon\left(\Upsilon^{\prime}, \Upsilon^{\prime \prime}\right) \rightarrow \gamma \mathrm{H}^{0}$, with a branching ratio larger according to eq. (1) (with $m_{\psi}$ replaced by $m_{\Upsilon}$ ). This gives, not counting the effect of the enhancement due to VEV's, $\operatorname{BR}\left(\Upsilon \rightarrow \gamma \mathrm{H}^{0}\right) \simeq$ $(2.1 \pm 0.3) \times 10^{-4}$, with similar results for $\Upsilon^{\prime}, \Upsilon^{\prime \prime}$. Interestingly, the effect of the VEV enhancement for charm (a factor of 10 in BR if the $\xi(2.2)$ is a Higgs

${ }^{\ddagger 3}$ In the calculations of $\mathrm{Hall}$ and Wise, the $\mathrm{H}^{0}$ was taken to be the pseudoscalar. However, the coupling of fermions to the scalar and pseudoscalar Higgs are not very different (although extra parameters are involved, see refs. $[6,7]$ ). Hence, we have assumed that the rate for $b \rightarrow s+$ scalar Higgs is similar to the results of ref. [10].

${ }^{74}$ It is interesting to note that in models with one Higgs doublet, the transition $\mathrm{b} \rightarrow \mathrm{s}+\mathrm{H}^{0}$ is in general substantially suppressed (see ref. [12]). One reason is that the strength of the Higgs - fermion coupling is fixed (i.e. $\eta=1$ ). However, in both the one and two doublet models, this rate is proportional to $m_{\mathrm{t}}^{4}$. So, if a light Higgs particle is found, the t-quark mass will be severely constrained. boson) can lead to either enhancement or suppression in $\Upsilon$ decay. In one class of models [6] (denoted type I) one VEV gives mass to all quarks (the other Higgs doublet is decoupled from the quark sector). Then, all $f \bar{f} \mathrm{H}^{0}$ couplings are enhanced by the same factor and one expects an enhanced rate, $\mathrm{BR}(\Upsilon \rightarrow \gamma \xi(2.2)) \simeq$ $2 \times 10^{-3}$. Presumably either by $\xi \rightarrow \mathrm{K}^{+} \mathrm{K}^{-}, \mathrm{K}_{\mathrm{S}} \mathrm{K}_{\mathrm{S}}$, or $\mu^{+} \mu^{-}$this should be observable. However, in a second class of models [7] (denoted type II) one VEV gives mass to up-type quarks $(u)$ and the other to downtype quarks (d). Then if the uū $\mathrm{H}^{0}$ coupling is proportional to an enhancement factor of $\eta$, the $\mathrm{d} \overline{\mathrm{d}} \mathrm{H}^{0}$ coupling is proportional to $\eta^{-}$and one expects $\mathrm{BR}(\Upsilon \rightarrow$ $\gamma \xi(2.2)) \simeq 2 \times 10^{-5}$, which may difficult to observe. ${ }^{\neq 5}$

Because of this situation, searching for $\xi(2.2)$ in $\Upsilon\left(\Upsilon^{\prime}, \Upsilon^{\prime \prime}\right)$ decay may not be able to confirm or deny the Higgs interpretation. [It should be added that in models where the ratio of VEV's is different from unity in order to give up-type and down-type quarks mass with similar couplings, one expects the Higgs coupling to the heavier up-type quarks to be reduced (since $g_{\mathrm{Hff}} \sim m_{\mathrm{f}} / v$ ) which is just the opposite of the situation here. If the Higgs interpretation for the $\xi(2.2)$ were to hold up, it would be difficult to understand how type II models could be relevant.]

(c) There are two $\mathrm{Z}^{0}$-decays where a very light $\mathrm{H}^{0}$ could show up in the next couple of years. First there is the well-known decay [13] $\mathrm{Z}^{0} \rightarrow \mathrm{Z}^{0} \mathrm{H}^{0}$ where one looks at the missing mass opposite the outgoing (virtual) $Z^{0}$. One can add up all the observable modes of virtual $Z^{0}\left(\mathrm{e}^{+} \mathrm{e}^{-}, \mu^{+} \mu^{-}\right.$, possibly jet pairs) to get a larger rate [14]. This may also be possible at a hadron collider by taking $Z^{0}$ 's produced with no recoil gluon jet. For very light $\mathrm{H}^{0}$ the rate is expected to be nearly $10^{-2}$ of the total $Z^{0}$ cross section $[15,16]$.

Second, if there are two or more neutral Higgs, as must occur with two or more doublets, then the decay $\mathrm{Z}^{0} \rightarrow \mathrm{H}_{1}^{0} \mathrm{H}_{2}^{0}$ is allowed and has a large branching ratio. ${ }^{\neq 6}$ If $\mathrm{H}_{2}^{0}$ is heavy its $\mu^{+} \mu_{-}^{-}$branching ratio might be small, so $c \bar{c}$ or $b \bar{b}$ (or even $\bar{t} \overline{\text { ) }}$ could dominate.

\$5 Strictly speaking, these remarks are relevant only for the pseudoscalar Higgs. The scalar Higgs-fermion couplings depend on parameters of the scalar potential in addition to $\eta$ leading to further uncertainty in the above predictions.

${ }^{\ddagger 6}$ Assuming $C P$ invariant couplings, $\mathrm{Z}^{0} \rightarrow \mathrm{H}_{1}^{0} \mathrm{H}_{2}^{0}$ implies that one of the neutral Higgs in the final state is a scalar $\left(0^{++}\right)$ and the other is a pseudoscalar $\left(0^{-+}\right)$. 
Then one would expect events with (say) a $\mu^{+} \mu^{-}$ from $\mathrm{H}_{1}^{0}$ opposite a heavy quark jet pair from $\mathrm{H}_{2}^{0}$. The precise branching ratio [8] depends on the Higgs potential and the mixing parameters which determine the mass eigenstates, but in the best case one can have $\Gamma\left(\mathrm{Z}^{0} \rightarrow \mathrm{H}_{1}^{0} \mathrm{H}_{2}^{0}\right) / \Gamma\left(\mathrm{Z}^{0} \rightarrow \mu^{+} \mu^{-}\right) \gtrsim 3$ before phase space suppression.

(d) Another possibility is the process $\bar{p} \overrightarrow{\mathrm{p}} \rightarrow \mathrm{W}^{ \pm} \mathrm{H}^{0}+$ $X$ which proceeds via $W^{ \pm} \rightarrow W^{ \pm} H^{0}$ and occurs at a rate of about $1 \%$ of the total $W$ cross section [15]. It is interesting to note that the $\xi(2.2)$ is within a resonance width of the $W$. This enhances the production rate for a light Higgs because the virtual $W$ is very nearly onshell. Thus, it may be possible to find a light Higgs boson at the $\bar{p} \overline{\mathrm{p}}$ collider.

(e) At the FNAL fixed target machine it may be possible to produce and detect a light $\mathrm{H}^{0}$, and 2.2 $\mathrm{GeV}$ is a good place to look. Higgs bosons may be produced by hard scattering of constituent quarks or gluons. In the case of $q \bar{q} \rightarrow \mathrm{H}^{0}$, the cross sections are very small because of the small $\mathrm{q} \overline{\mathrm{q}} \mathrm{H}^{0}$ coupling proportional to a light ( $u$ or $d$ ) quark mass. One does better if the constituent process is $\bar{s} \rightarrow \mathrm{H}^{0}$. This process could be observable in $\mathrm{KN} \rightarrow \mu \mu \mathrm{X}$ where one of the strange quarks is a valence quark. In ref. [6], we showed that (for an experimental resolution of 10 $\mathrm{MeV}$ ) the Higgs signal to Drell-Yan background would be $0.3 \eta^{2}$ where $\eta$ is the enhancement factor for the $\mathrm{H}^{0} \overline{\mathrm{s}}$ coupling. ${ }^{\neq 7}$ Hence, for $\eta^{2} \approx 10$, we find a possible signal-to-noise ratio of $3: 1$ for finding a Higgs boson at $2 \mathrm{GeV}$.

One can also produce Higgs bosons by gluongluon fusion through an intermediate quark loop [17]. Again, the enhancement factor $\eta^{2} \approx 10$ helps to increase the rate by a factor of 10 or 5 (for type $I$ and II models, respectively). This leads to $\mathrm{d} \sigma /\left.\mathrm{d} y\right|_{y=0} \approx 1$ nb (at $E_{\text {lab }}=1 \mathrm{TeV}$ ) for a $2 \mathrm{GeV}$ Higgs boson. Folding in a $10 \% \mu^{+} \mu^{-}$branching ratio, the signal as compared with the Drell-Yan background is marginal but perhaps not impossible to observe. Furthermore, it might be possible to search for other exclusive modes of the Higgs, for example in $\mathrm{pp} \rightarrow \mathrm{K}_{\mathrm{S}} \mathrm{K}_{\mathrm{S}}+\mathrm{X}$.

(f) One further possibility is to make use of LEAR, the high luminosity $\bar{p} \bar{p}$ facility which operates in the

\footnotetext{
${ }^{\ddagger 7}$ Note that in type Il models, an enhanced $\mathbf{H}^{0} \mathrm{c} \overline{\mathrm{c}}$ coupling implies that the $\mathrm{H}^{0} \mathrm{~s} \bar{s}$ coupling is suppressed so that its process would not be likely to uncover the Higgs boson in these models.
}

$2 \mathrm{GeV}$ region. There have been discussions of the possibility of operating LEAR as a minicollider at the $\psi$ mass [18]. (The luminosity on the $\psi$ with present technology was estimated [18] to be about $10^{29}$ $\mathrm{cm}^{-2} \mathrm{~s}^{-1}$.) If the luminosity could be increased, such a machine would be very useful in studying rare $\psi$ decays. For example, in ref. [19], it is found that $\sigma(\mathrm{p} \overline{\mathrm{p}} \rightarrow \psi) \approx 5 \mu \mathrm{b}$ which would imply over ten $\xi(2.2)$ $\rightarrow \mathrm{K}^{+} \mathrm{K}^{-}$events produced per hour given a luminosity of $10^{31} \mathrm{~cm}^{-2} \mathrm{~s}^{-1}$.

\section{Implications for current ideas. If any Higgs par-} ticles were found it would, of course, be of the greatest important for the development of particle physics. A very light Higgs with a mass in the few $\mathrm{GeV}$ range would strongly constrain ideas since it does not arise naturally in many approaches. As we discussed above, only approaches equivalent to a model with more than a single Higgs doublet could give the required branching ratio enhancement.

A priori, the Higgs masses are free parameters in almost all approaches. One exception occurs in a model by Coleman and Weinberg [20] who set the tree-level Higgs mass to zero and generate spontaneous symmetry breaking by radiative corrections. In the one-Higgs doublet model, the physical Higgs boson gains a radiative mass equal to $m_{\mathrm{H}}=2 v(2 C)^{1 / 2}$ [where $C$ is given by eq. (2)] which, ignoring fermion masses, is about $10 \mathrm{GeV}$. In multi-Higgs models, among the numerous physical Higgs bosons, there is one scalar Higgs which gains a mass radiatively given by the same formula as above [21]. It is amusing to note that if a fermion (t-quark?) exists which is massive enough, then the value of $C$ can be reduced sufficiently so that $m_{\mathrm{H}}=$ $2.2 \mathrm{GeV}$ ! Thus, such a light Higgs boson mass may not be incompatible with the Coleman-Weinberg mechanism.

In supersymmetric theories at least two Higgs doublets are required to give mass to up and down type quarks [22], so a Higgs interpretation for $\xi(2.2)$ could be accommodated. However, the ratio of VEV's cannot be easily made to go in the direction to enhance the charm coupling - it naturally goes the other way (due to a heavy top quark) [23]. In addition, in many models, the mass scale for $\mathrm{H}^{0}$ is $m_{\mathrm{W}}$ rather than $\mathrm{O}(1 \mathrm{GeV})$ (note, however, the model of Kounnas et al. [23] where a light Higgs boson in the $3-6 \mathrm{GeV}$ range is predicted). Consequently, while supersymmetric 
theories technically could deal with $\xi(2.2)$, they could not do so without considerable manipulation, and none of the present approaches to models are of the necessary form.

The situation in technicolor [24] approaches ${ }^{\ddagger 8}$ is subtle. Light neutral states are predicted [26] around $2.2 \mathrm{GeV}$. All the light states are pseudoscalars while $\xi(2.2)$ decays to $\mathrm{K}_{\mathrm{S}} \mathrm{K}_{\mathrm{S}}$ which has positive parity. However, parity is not conserved in the transition from technifermions to ordinary fermions, so perhaps an initial pseudoscalar can decay to a positive parity final state. One of us has recently analyzed this situation [27]. The answer is that a self-conjugate initial pseudoscalar, $\overline{\mathrm{Q}} \gamma_{5} \mathrm{Q}$, formed by a technifermion $\mathrm{Q}$, cannot decay into an ss scalar final state if $C P$ is conserved. Thus no simple technicolor model could accommodate $\xi(2.2)$ in spite of the mass value. A more complicated model where the initial pseudo was not self-conjugate, e.g. $\overline{\mathrm{S}} \gamma_{5} \mathrm{D}$, could give a scalar final state and accommodate $\xi(2.2)$. It immediately requires [27], by analogy with $\mathrm{K}_{\mathrm{S}}-\mathrm{K}_{\mathrm{L}}$ system, another state of opposite $C P$ quite near in mass, which could appear in $\mathrm{K}^{*} \overline{\mathrm{K}}$. Such a model would have as further consequences the appearance of several other neutral pseudoscalar states on the same scale of a few $\mathrm{GeV}$, and of more charged states in the 8-20 $\mathrm{GeV}$ range. The size of these masses is constrained if the $\xi(2.2)$ is a technicolor state since then one has an upper limit on a value for contributions to its mass besides those from standard model interactions. In summary, while interpreting $\xi(2.2)$ as a technicolor state is not easy or natural, it is not excluded. However, if the technicolor interpretation were correct, a number of other related neutral and charged states nearby in mass must exist.

It is worth emphasizing that if the $\xi(2.2)$ turns out to be the Higgs boson, it would be possible to devise tests to rule out the technicolor approach. Technicolor models cannot coexist with light scalar Higgs bosons (in the technicolor scenario, these particles lie in the range $100-1000 \mathrm{GeV}$ ). As argued above, the light pseudoscalars of technicolor could exhibit scalar-like couplings to fermions but such models have

$\neq 8$ By technicolor approaches, we mean to include extended technicolor (ETC) interactions (ref. [25]) which are needed to obtain masses for the light fermions. These ETC interactions also lead to Yukawa couplings of the fermions to the light pseudoscalar states. observable consequences. One definitive way to rule out technicolor would be to observe the decay $\mathrm{Z}^{0} \rightarrow$ $\mathrm{H}_{1}^{0} \mathrm{H}_{2}^{0}$ which would imply the existence of at least one light scalar Higgs.

\section{Comments. If any state can be interpreted as a} Higgs particle it must have a number of properties and satisfy a number of conditions; we have summarized those of which we are aware. As we have hinted, even if $\xi(2.2)$ has a narrow width, spin zero, and a significant $\mu^{+} \mu^{-}$decay, it will be difficult to sustain a Higgs interpretation in the context of currently known extensions of the standard model. The $\xi(2.2)$ cannot be a Higgs scalar in the standard model with a single doublet because of the large branching ratio in $\psi \mathrm{de}$ cay, and perhaps because of its light mass. If the $\xi(2.2)$ is a Higgs scalar in a multi-Higgs model, then it is surprising that it has not appeared in b-quark decays. However, the fact that it is not accommodated easily in any present approach to physics beyond the standard model may say more about the approaches than about the $\xi(2.2)$. But because of the great significance of Higgs physics, every effort should be made to test whether $\xi(2.2)$ or any other future candidate could be a Higgs particle.

We gratefully acknowledge Michael Barnett, Kevin Einsweiler, John Ellis, Ricardo Flores, Larry Hall, Paul Langacker, Peter Ratoff and Jonathan Rosner for useful discussions. One of us (G.L.K.) thanks the Aspen Center for Physics for its hospitality while some of this work was being done.

\section{References}

[1] S.L. Glashow, Nucl. Phys. 22 (1961) 579; S. Weinberg, Phys. Rev. Lett. 19 (1967) 1264; A. Salam, in: Proc. 8th Nobel Symp. on Elementary particle theory, relativistic groups and analyticity, ed. N. Svartholm (Almquist and Wiksells, Stockholm, 1968) p. 367

[2] G.L. Kane, in: Gauge theories in high energy physics, Les Houches Summer School Proc., Vol. 37, eds. M.K. Gaillard and R. Stora (North-Holland, Amsterdam, 1983);

K. Lane, in: Proc. 1982 DPF Summer Study on Elementary particle physics and future facilities, eds. R. Donaldson, R. Gustafson and F. Paige (American Physical Society, New York, 1983). 
[3] Walter Toki, invited talk, 1983 SLAC Summer Institute on Particle Physics.

[4] F. Wilczek, Phys. Rev. Lett. 39 (1977) 1304.

[5] A.D. Linde, JETP Lett. 23 (1976) 64; Phys. Lett. 70B (1977) 306; S. Weinberg, Phys. Rev. Lett. 36 (1976) 294.

[6] H.E. Haber, G.L. Kane and T. Sterling, Nucl. Phys. B161 (1979) 493.

[7] J.F. Donoghue and L.-F. Li, Phys. Rev. D19 (1979) 945; B. McWilliams and L.F. Li, Nucl. Phys. B 179 (1981) 62 .

[8] H. Hüffel and G. Pócsik, Z. Phys. C8 (1981) 13; G. Pócsik and G. Zsigmond, Z. Phys. C10 (1981) 367; Phys. Lett. 112B (1982) 157.

[9] N.-P. Chang and C.A. Nelson, Phys. Rev. Lett. 40 (1978) 1617 ;

T.L. Trueman, Phys. Rev. D18 (1978) 3423.

[10] M.B. Wise, Phys. Lett. 103B (1981) 121; L.J. Hall and M.B. Wise, Nucl. Phys. B1 87 (1981) 397.

[11] S. Stone, invited talk, 1983 Intern. Symp. on Lepton and photon interactions at high energies (Cornell Univ., August 1983).

[12] R.S. Willey and H.L. Yu, Phys. Rev. D26 (1982) 3086; B. Grzadkowski and P. Krawczyk, Z. Phys. C18 (1983) 43.

[13] J.D. Bjorken, in: Proc. SLAC Summer Institute on Particle physics, SLAC-198 (1976) p. 1; J. Finjord, Phys. Scr. 21 (1980) 143.

[14] V. Barger, F. Halzen and W.Y. Keung, Phys. Lett. 110B (1982) $322 ; 117 \mathrm{~B}(1982) 476(\mathrm{E})$.

[15] S.L. Glashow, D.V. Nanopoulos and A. Yildiz, Phys. Rev. D 18 (1978) 1724;

J. Finjord, G. Girardi and P. Sorba, Phys. Lett. 89B (1979) 99.

[16] J. Ellis, M.K. Gaillard and D.V. Nanopoulos, Nucl. Phys. B106 (1976) 292;

B.W. Lee, C. Quigg and H.B. Thacker, Phys. Rev. D 16 (1977) 1519.

[17] H.M. Georgi, S.L. Glashow, M.E. Machacek and D.V. Nanopoulos, Phys. Rev. Lett. 40 (1978) 692.

[18] U. Gastaldi and K. Kilian, in: Proc, of the Kaon Factory Workshop, ed. M.K. Craddock, TRIUMF publication TRI-79-1 (1979) p. 43;

U. Gastaldi and R. Klapisch, in: From nuclei to particles: Proc. 1980 Intern. School of Physics Enrico Fermi,
79 th Course, ed. A. Molinari (North-Holland, Amsterdam, 1982) p. 462.

[19] R.N. Cahn and M. Suzuki, Phys. Lett. 93B (1980) 179.

[20] S. Coleman and E. Weinberg, Phys. Rev. D7 (1973) 1888.

[21] E. Gildener and S. Weinberg, Phys. Rev. D13 (1976) 3333;

K. Inoue, A. Kakuto and Y. Nakano, Prog. Theor. Phys. 63 (1980) 234;

R.A. Flores and M. Sher, Ann. 148 (1983) 95.

[22] E. Witten, Nucl. Phys. B188 (1981) 513;

S. Dimopoulos and H. Georgi, Nucl. Phys, B193 (1981) 150 ;

N. Sakai, Z. Phys. C11 (1981) 153.

[23] K. Inoue, A. Kakuto, H. Kamatsu and S. Takeshita, Prog. Theor. Phys. 67 (1982) 1889 ;

L.E. Ibanez, Phys. Lett. 118B (1982) 73; Nucl. Phys. B21 8 (1983) 514 ;

L. Alvarez-Gaume, J. Polchinski and M.B. Wise. Nucl. Phys. B221 (1983) 495;

J. Ellis, J.S. Hagelin, D.V. Nanopoulos and K. Tamvakis, Phys. Lett. 125B (1983) 275;

L.E. Ibanez and C. Lopez, Phys. Lett. 126B (1983) 54;

[25] C. Kounnas, A.B. Lahanas, D.V. Nanopoulos, M. Quiros, Phys. Lett. 132B (1983) 95.

[24] S. Wein berg, Phys, Rev. D13 (1976) 974; D19 (1979) 1277 ;

L. Susskind, Phys. Rev. D20 (1979) 2619;

E. Farhi and L. Susskind, Phys. Rep. 74C (1981) 277.

[25] S. Dimopoulos and L. Susskind, Nucl. Phys. B155 (1979) 237;

E. Eichten and K. Lane, Phys. Lett. 90B (1980) 125.

[26] M.A.B. Beg, H.D. Politzer and P. Ramond, Phys. Rev. Lett. 43 (1979) 1701;

S. Dimopoulos, Nucl. Phys. B168 (1980) 69;

M.E. Peskin, Nucl. Phys. B175 (1980) 197;

J.P. Preskill, Nucl. Phys. B177 (1981) 21;

S. Dimopoulos, S. Raby and G.L. Kane, Nucl. Phys.

B 182 (1981) 77;

S. Chadha and M.E. Peskin, Nucl. Phys. B185 (1981) 61; B187 (1981) 541;

P. Binétruy, S. Chadha and P. Sikivie, Phys. Lett. 107B (1981) 425; Nucl. Phys. B207 (1982) 505.

[27] H.E. Haber, Can the $\xi(2.2)$ be a techni-pion?, SLACPUB-3193 (August, 1983). 